\title{
GENETIC TRANSFORMATION OF Melia azedarach L., USING Agrobacterium MEDIATED TRANSFORMATION
}

\author{
Arif Nirsatmanto ${ }^{1}$ and Koichiro Gyokusen ${ }^{2}$
}

\begin{abstract}
This study was subjected to observe the possibility of introducing specific foreign genes into Melia azedarach L., using Agrobacterium mediated transformation. Agrobacterium tumefaciens used in this study consisted of strains of EHA105 (vector plasmid pBIsGFP) and EHA105 (vector plasmid pBsGFP) to observe the possibility of introducing genes, and strains of EHA101 (vector plasmid pIG121-Hm) and LBA4404/ferritin (vector plasmid pBG-1) to observe the shoot organogenesis after genes transformation. Explants were collected from one $\mathrm{cm}$ in length excised stem of in-vitro plantlets. The results of the study showed that genetic transformations of M. azedarach could be potentially developed using Agrobacterium tumefaciens strains : EHA105 (pBIsGFP or pBsGFP), EHA101 (pGI121-Hm) and LBA4404/ferritin (pBG-1). The expression of GFP (green fluorescence protein) signal worked successfully in this transformation with $40 \%$ of transformation rate for pBIsGFP and $20 \%$ for pBsGFP. The application of Agrobacterium strains of EHA101 (pIG121-Hm) and LBA4404/ferritin (pBG-1) which contained specific gene of kanamycin resistance and iron accumulation for plant growth improvement showed that adventitious shoot was well induced and elongated on the rate of $30-60 \%$ of explants after genes transformation.
\end{abstract}

Keywords: Melia azedarach L., in-vitro culture, genetic transformation, Agrobacterium tumefaciens

\section{INTRODUCTION}

Genetic engineering through genetic transformation has played important role for breeding purposes in advance tree improvement program. It is due to the success of genetic transformation which may introduce such novel genetic character resistance to disease, insect, and herbicide. This becomes a very important part of producing new improved variety in forest tree species. The success of genetic transformation requires a high regenerative system of organogenesis, because of low efficiency of transformation due to kind of methods that was applied to introduce foreign gene into plants, such as using Agrobacterium, particle bombardment or electroporation (Kawazu et al., 1996).

Introducing specific foreign genes into plant through genetic engineering could increase the growth of transformed plant due to increasing the content of iron inside plant metabolism (Goto et al., 2000). Genetic transformation technology has proved the benefit of transformed plant for increasing productivity. However, the considerable progress of genetic transformation study for forest tree is still quite limited which are restricted to poplar and eucalypts (Cheliak and Rogers, 1990; Harcourt et al., 2000). To date, study on genetic transformation for Melia azedarach L., has not yet been reported.

\footnotetext{
${ }^{1}$ Forest Biotechnology and Tree Improvement Research Center, Yogyakarta, Indonesia

${ }^{2}$ Faculty of Agriculture, Kyushu University, Kyushu, Japan
} 
Melia azedarach L., (Persian Lilac, Ghora Neem) is one of the important species in Meliaceae family which primary native to the Orient, especially in Iran, China, India, Japan, and naturalized in many sub-tropical region of the world (Kelvey, 1928). M. azedarach is high priority multi-purposes tree due to capability to produce valuable timber resistance to termites, fodder, green manure, and oil from seed. This species also contains therauptic, insecticidal properties and some several compounds of limonoids (Itokawa et al., 1995; Huang et al., 1996).

In term of economic benefits of $M$. azedarach L., improving quality by applying the genetic transformation technology is necessary. It is supported with the success of shoot organogenesis of this species which are a prerequisite matter for the success of genetic transformation (Nirsatmanto, 2002). However, to date, study on genetic transformation for M. azedarach $\mathrm{L}$ has not yet been reported. In the present study, we observed the possibility of introducing specific foreign genes into M. azedarach using Agrobacterium mediated transformation. This method was selected due to it has become widely used for genetic transformation, and it also showed higher gene transfer efficiency than using non-bacterium methods (Muramatsu et al., 1998).

\section{MATERIAL AND METHODS}

\section{A. Explants}

Explants used in this study were collected from in-vitro plantlets of M.azedarach. Stems (with no axillary bud) were excised from the plantlets and cut into about one $\mathrm{cm}$ in length as explants.

\section{B. Agrobacterium strains}

In the first stage, the GFP (Green Fluorescence Protein) of A. tumefaciens strains of EHA105 (vector plasmid pBIsGFP) and EHA105 (vector plasmid pBsGFP) were used to observe possibility of introducing genes into $M$. azedarach. Thereafter, the $A$. tumefaciens strains of EHA101 (vector plasmid pIG121-Hm) and LBA4404/ferritin (vector plasmid pBG-1) were applied to observe the shoot organogenesis after transformation (Otani et al., 1998; Goto et al., 1998). Before co-cultivation of the bacteria with the explants, A. tumefaciens strains of EHA101 and LBA4404 were grown overnight at $25^{\circ} \mathrm{C}$ in LB (Luria Bertani medium)-antibiotic agar medium containing $100 \mathrm{mg} / \mathrm{l} \mathrm{kanamycin} \mathrm{and} 20 \mathrm{mg} / \mathrm{lof}$ rifampicin. In the case of two $A$. tumefaciens strains of EHA105, they were grown in absence of rifampicin.

The signal of pBIsGFP will only detect the inserted genes into plant without performing signal for any other organs, while the signal of pBsGFP will detect not only the inserted genes, but also agrobacterium that might be still remained in explants after cocultivation. The EHA101 and LBA4404/ferritin are the genes with a function to increase the kanamycin resistance and the accumulation of iron content in plant for increasing the growth. (Hood et al., 1986; Goto et al., 1998) 


\section{Transformation procedure}

Explants were immersed with agitation for 2 minutes in MS (Murashige and Skoog's) medium containing overnight grown bacteria that was grown in overnight, supplemented with 2\% sucrose, BAP (6-bensylaminopurine) $1 \mu \mathrm{M}$, NAA ( $\alpha$-naphthaleneacetic acid) $0.01 \mu \mathrm{M}$ (Nirsatmanto, 2002), and $10 \mathrm{mg} / \mathrm{l}$ of acetosyringone. The immersed explants were then blotted drying using sterile filter papers and transferred into solid MS medium supplemented with the same concentration of hormone, sucrose, and acetosyringone for co-cultivation. The co-cultivation culture was maintained under $28^{\circ} \mathrm{C}$ with 0 light intensity (dark).

After three days of co-cultivation, explants were rinsed with agitation for three times ( 2 minutes interval) using $500 \mathrm{mg} / \mathrm{l}$ of carbenicilin. After blotted drying using sterile filter papers, explants were transferred to selection medium containing MS medium supplemented with BAP $1 \mu \mathrm{M}$, NAA $0.01 \mu \mathrm{M}$, sucrose 2\%, kanamycin $100 \mathrm{mg} / \mathrm{l}$, rifampicin $20 \mathrm{mg} / \mathrm{l}$ and carbenicilin $500 \mathrm{mg} / \mathrm{l}$ for agrobacterium strains of EHA101 and LBA4404, and without riffanpicin for the two of EHA105. Investigation was done every week to observe the differentiation of transformed shoots. In the case of strain of EHA105 (pBIsGFP and pBsGFP), observation was done using the fluorescence photographs microscope. Gene transformations for strains of EHA101 (pIG121-Hm) and LBA4404 / ferritin (pBG-1) were done twice (hereinafter referred as Research 1 and Research 2).

\section{RESULTS AND DISCUSSION}

\section{A. Strains of EHA105 (vector plasmid pBIsGFP) and EHA105 (vector plasmid pBsGFP)}

Observation using Fluorescence Photographs of Nikon's P-FLA Fluorescence Attachment with the stereoscopic microscope SMZ800 showed that the expression of EHA105 (pBIsGFP and pBsGFP) occurred in the segment of stem explants of M. azedarach after two weeks of culturing into selection medium. The bright fluorescent signal which indicated the inserted of gene transformation into explants was shown in Figure 1.

The expression of $\mathrm{pBIsGFP}$ into stem explants indicated that transformation rate was higher than that of pBsGFP: $40 \%$ for pBIsGFP and $20 \%$ for pBsGFP (Table 1). It means that $A$. tumefaciens strain EHA105 with vector plasmid pBIsGFP seemed to be more effective for mediated transformation of $M$. azedarach than that EHA105 with vector plasmid $\mathrm{pBsGFP}$. Moreover, the expression of $\mathrm{pBsGFP}$ itself is probably showed the signal of agrobacterium which might be still remained on surrounding explants after co-cultivation. 


\section{$A$}
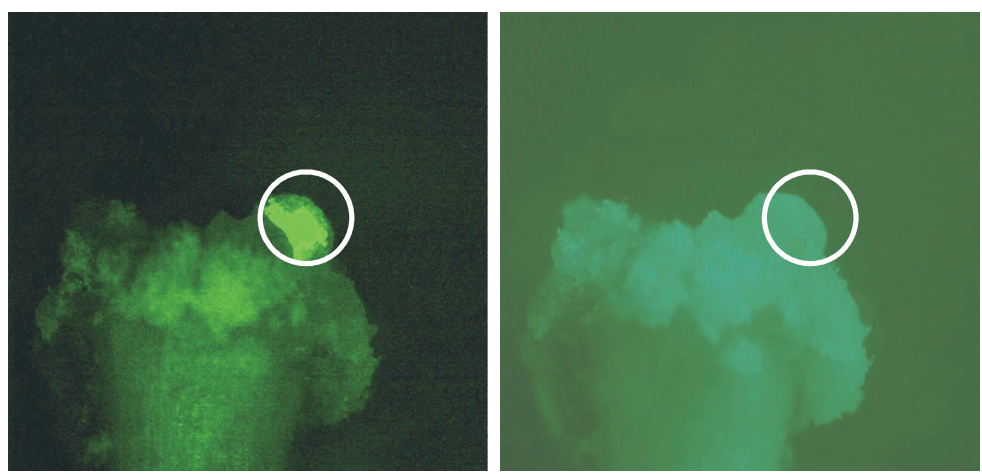

$B$
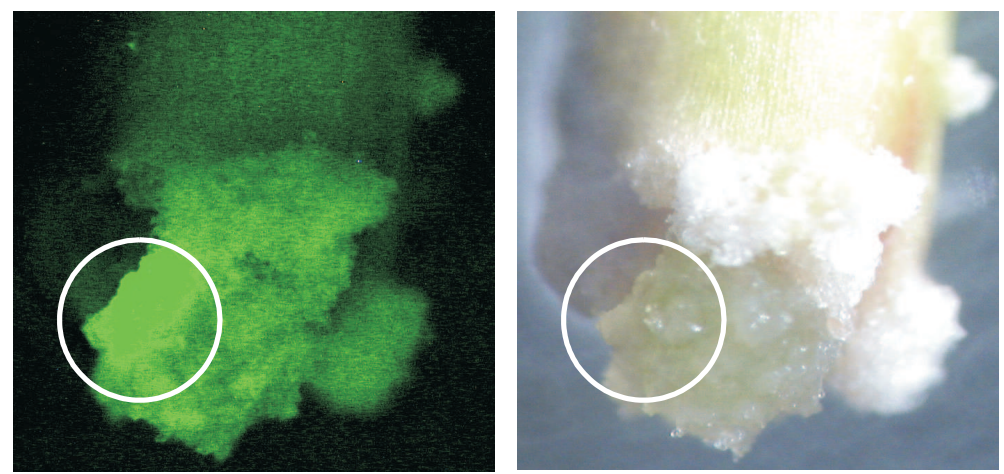

Figure 1. The expression of EHA105 (pBIsGFP and pBsGFP) into stem explants of $M$. azedarach after two weeks culturing on selection medium: $(A)$ the inserted gene transformed using strain of EHA105 (pBIsGFP), (B) the inserted gene transformed using strain of EHA105 (pBsGFP). Remarks : The clearly green colours (circle) is bright fluorescent signal indicating the inserted of gene transformation. The right hand side pictures are the whole explants with detail position of inserted gene.

Table 1. The effects of $A$. tumefaciens strains of EHA105 (pBIsGFP and pBsGFP) on transformation rate of $M$. azedarach stem explants after culturing into selection medium containing BAP $1 \mu \mathrm{M}$, NAA $0.01 \mu \mathrm{M}$, kanamycin $100 \mathrm{mg} / \mathrm{l}$, and carbenicilin $500 \mathrm{mg} / \mathrm{l}$.

\begin{tabular}{lccc}
\hline Agrobacterium strain & $\begin{array}{c}\text { Number of } \\
\text { explants }\end{array}$ & $\begin{array}{c}\text { Number of explants } \\
\text { showing the expression of GFP } \\
\text { after transformation }\end{array}$ & $\begin{array}{c}\text { Frequency of } \\
\text { transformation } \\
(\%)\end{array}$ \\
\hline EHA105 (pBIsGFP) & 10 & 4 & 40 \\
EHA105 (pBsGFP) & 10 & 2 & 20 \\
\hline
\end{tabular}


The expression of GFP signal in this study indicated that foreign gene has been potentially introduced into M. azedarach explants. However, this study is the first application of GFP transformation system for $M$. azedarach. Therefore further study is still necessary to improve the efficiency of GFP system for developing $M$. azedarach genetic transformation.

\section{B. Strains of EHA101 (vector plasmid pIG121-Hm) and LBA4404/ferritin (vector plasmid pBG-1)}

The growth of adventitious shoot of $M$. azedarach after transformation using $A$. tumefaciens strains of EHA101 and LBA4404 showed that the first differentiated adventitious shoots were observed after three weeks culturing in selection medium containing $100 \mathrm{mg} / \mathrm{lof}$ kanamycin, $20 \mathrm{mg} / \mathrm{l}$ of rifampicin and $500 \mathrm{mg} / \mathrm{l}$ of carbenicilin. Within 10 weeks (Research 1) and 6 weeks (Research 2) of culture transformation, the differentiated adventitious shoot rates decreased with the time of culture for $A$. tumefaciens strain EHA101: 65\% in 6 weeks and $45.5 \%$ in 10 weeks. On the other hand, the rates of strain Lb4404 slightly increased with the time: $35.7 \%$ in 6 weeks and $44.4 \%$ in 10 weeks (Table.2). Goto et al. (2000) reported that higher concentration of kanamycin $(100 \mathrm{mg} / \mathrm{l})$ and longer growth period was required for increasing selection transformation using LBA4404 / ferritin.

Table 2. The effects of $A$. tumefaciens strains of EHA101 (pIG121-Hm) and LBA4404 (pBG-1) on transformation rate of $M$. azedarach stem explants after culturing into selection medium containing BAP $1 \mu \mathrm{M}$, NAA $0.01 \mu \mathrm{M}$, kanamycin $100 \mathrm{mg} / \mathrm{l}$, rifampicin $20 \mathrm{mg} / \mathrm{l}$ and carbenicilin $500 \mathrm{mg} / \mathrm{l}$.

\begin{tabular}{lccc}
\hline \multicolumn{1}{c}{ Agrobacterium strain } & $\begin{array}{c}\text { Number of } \\
\text { explants }\end{array}$ & $\begin{array}{c}\text { Number of } \\
\text { differentiated shoots explants } \\
\text { after transformation }\end{array}$ & $\begin{array}{c}\text { Frequency of } \\
\text { transformation } \\
(\%)\end{array}$ \\
\hline Research 1 & 10 & 45.5 \\
EHA101 (pIG121-Hm) & 22 & 8 & 44.4 \\
LB4404 (pBG-1) & 18 & 26 & 65 \\
\hline Research 2 & 40 & 10 & 35.7 \\
EHA101 (pIG121-Hm) & 28 & & \\
LB4404 (pBG -1) & & 26 & \\
\hline
\end{tabular}

Note: The data on Research 1 were collected after 10 weeks, while the data on Research 2 were collected after 6 weeks culturing on selection medium.

The observation on differentiated adventitious shoots grown in selection medium showed that the rates of shoots differentiation were slow (Figure 2). Shoot growth inhibition in this study might be due to the effects of the antibiotic of kanamycin, rifampicin and carbenicilin that were used during transformation. 

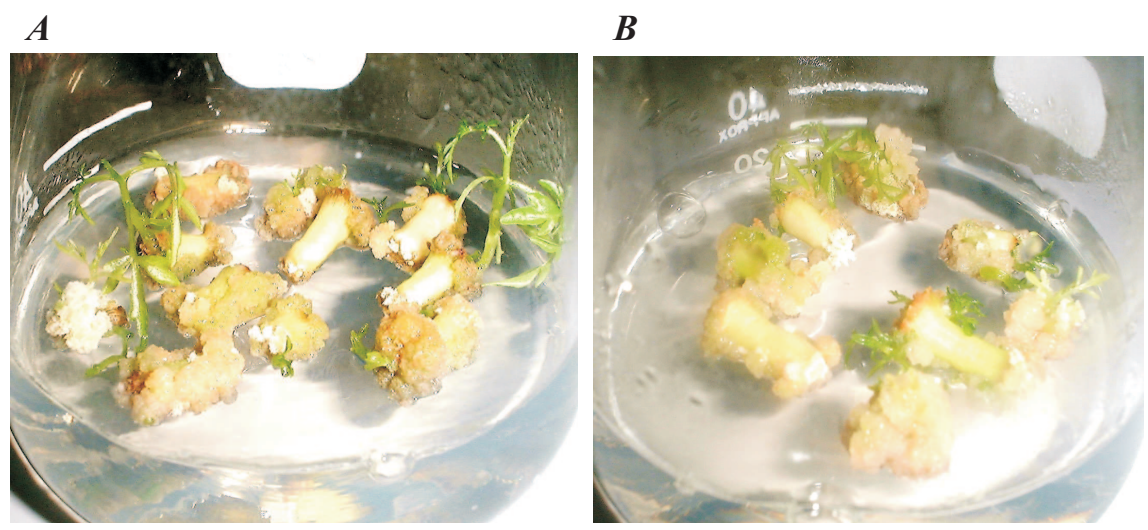

Figure 2. The growth of differentiated adventitious shoot of $M$. azedarach for kanamycin resistance after transforming using $A$. tumefaciens: (A) transformed using EHA101 (pG121-Hm),(B) transformed using strain of LBA4404 (pBG-1). Remarks : The first transformed shoots were differentiated after three weeks culture on selection medium containing BAP $1 \mu \mathrm{M}$, NAA $0.01 \mu \mathrm{M}$, kanamycin $100 \mathrm{mg} / \mathrm{l}$, riffanpicin $20 \mathrm{mg} / \mathrm{l}$, and carbenicilin $500 \mathrm{mg} / \mathrm{l}$. Photos were taken on six weeks culture.

The observation was done in the initial stage of transformed shoots differentiation. Therefore to confirm whether the gene was clearly transformed into new plantlets, the transformed adventitious shoots should be analyzed later by excising and re-culturing into the same selection medium and verified using a technique of DNA molecular, such as PCR or Southern blotted analysis.

\section{CONCLUSIONS}

1. The results of the study showed that the genetic transformations of $M$. azedarach could be potentially developed using mediated transformation of $A$. tumefaciens strains : EHA105 (vector plasmid pBIsGFP or pBsGFP), EHA101 (vector plasmid pGI121-Hm) and LBA4404/ferritin (vector plasmid pBG-1), with selection marker resistance to kanamycin.

2. Detection of the possibility for introducing foreign gene into $M$. azedarach explants using GFP agrobacterium strains of EHA105 binary vector $\mathrm{pBIsGFP}$ and $\mathrm{pBs}$ GFP showed that the expression GFP signal worked successfully with transformation rate of $40 \%$ for pBIsGFP and $20 \%$ for pBsGFP. It indicated that foreign gene could be potentially introduced into $M$. azedarach explants.

3. The application of agrobacterium strains of EHA101 (pIG121-Hm) and LBA4404/ferritin (pBG-1) mediated transformation which contains specific gene for plant growth improvement (kanamycin resistance and iron accumulation) showed that adventitious shoot was well induced and elongated on $30-60 \%$ of $M$. azedarach explants after transformation. 


\section{Acknowledgement}

This study was done in the Silviculture Lab., Fac. of Agriculture, Kyushu University Japan as a part of author's study in the topic of "Development of shoot organogenesis system of Melia azedarach L., and its application for genetic transformation". Authors express sincerely gratitude to all members of the Silviculture Lab., Fac. of Agriculture, Kyushu University.

\section{REFERENCES}

Cheliak, W. M. and D. L. Rogers. 1990. Integrating biotechnology into tree improvement programs. Canadian Journal of Forest Research 20:452-463.

Goto, F., T. Yoshihara, and H. Saiki. 1998. Iron accumulation in tobacco plants expressing soybean ferritin gene. Transgenic Research 7:173-180.

Goto F., T. Yoshihara, and H. Saiki. 2000. Iron accumulation and enhanced growth in transgenic lettuce plants expressing the iron-binding protein ferritin. Theoretical and Applied Genetic 100:658-664.

Harcourt, R. L., J. Kyozuka, R.B. Floyd, K.S. Bateman, H. Tanaka, V. Decroocq, D. J. Lewellyn, X. Zhu, W. J. Peacock, and E. S. Dennis. 2000. Insect-and herbicideresistant transgenic eucalypts. Molecular Breeding 6:307-315.

Hood, E. E., G. L. Helmer, R. T. Fraley, and M. D. Chilton. 1986. The hypervirulence of Agrobacterium tumefaciens A281 is encoded in a region of pTiBo542 outside of T-DNA. Journal of Bacteriol Bacteriology 168:1291-1301.

Huang, R. C., K. Tadera, F. Yagi, Y. Minami, H. Okamura, T. Iwagawa, and M. Nakatani. 1996. Limonoids from Melia azedarach. Phytochemistry 43:581-583.

Itokawa, H., Z. Qiao, C. Hirobe, and K. Takeya. 1995. Cytotoxic limonoids and tetranortriterpenoids from Melia azedarach. Chemical and Pharmaceutical Bulletin 43:1171-1175.

Kawazu, T., K. Doi, Y. Tatemichi, K. Ito, and M. Shibata. 1996. Regeneration of transgenic plants by nodule culture system in Eucalyptus camaldulensis. In: M. J. Dieters, A. C. Matheson, D. G. Nikles, C. E. Harwood, and S. M. Walker (Eds.), Tree Improvement for Sustainable Tropical Forestry. Proceedings of the QFRI-IUFRO Conference, Caloundra, Queensland, Australia, 27 October-1 November. 492-497 pp.

Mc Kelvey, S. D. 1928. The Lilac : a monograph. The Mac Milan Company. New York. 
Journal of Forestry Research Vol. 4 No. 1, March 2007: 1 - 8

Muramatsu, T., A. Nakamura, and H. M. Park. 1998. In vivo electroporation: A powerful and convenient means of non-viral gene transfer to tissues of living animal (Review). International Journal of Molecular Medicine 1:55-62.

Nirsatmanto, A. 2002. Development of shoot organogenesis system of Melia azedarach L., and its application for genetic transformation. Master Thesis. Kyushu University, Japan.

Otani, M., T. Shimada, T. Kimura, and A. Saito. 1998. Transgenic plant poduction from embryogenic callus of sweet potato (Ipomoea batatas Lam.) using Agrobacterium tumefaciens. Plant and Biotechnology 15:11-16. 